\section{International Scientific Journal Theoretical \& Applied Science}

p-ISSN: 2308-4944 (print) e-ISSN: 2409-0085 (online)

Year: 2018 Issue: 09 Volume: 65

Published: $29.09 .2018 \quad \underline{\text { http://T-Science.org }}$

SECTION 2. Applied mathematics.

Mathematical modeling.
Normahmad Ravshanov

Doctor of Technical Sciences, prof.,

Head of Laboratory,

Tashkent University of Information Technologies,

Tashkent, Uzbekistan

ravshanzade-09@mail.ru

Utkir Saidov

Senior Lecturer, Samarkand Branch of Tashkent University of Information Technologies,

Samarkand, Uzbekistan

Tursun Shafiyev

Assistant,

Bukhara State University,

Bukhara, Uzbekistan

\title{
DECISION OF THE DIRECT AND REVERSE PROBLEM OF THE TECHNOLOGICAL PROCESS OF SUSPENSION FILTERING
}

\begin{abstract}
The article deals with the solution of an actual problem related to the technological process of filtration and dewatering of liquid solutions from finely divided particles. Above mentioned technological process is realized during filtering and cleaning of chemical solutions, drinking water, pharmaceuticals, liquid fuel, public products, etc. For analysis, research, determination of the main parameters of the technological process, as well as modes of operation of filter units and support for management decision-making, a mathematical model has been developed, where it is possible to take into account the different operating conditions of the filter unit and the physicochemical properties of solutions.

The article notes that the study of the process of filtering a suspension, determining the main parameters of the process under consideration and their ranges of change for the purpose of managing it at functioning objects makes it difficult to collect data on the process, it takes a lot of time to perform a series of experiments in the laboratory that take a lot of labor and time and It is difficult to find the relationship between the parameters of the filter and the technological process for a limited experimental sample.

Taking into account the above, it can be said that the current task is to determine the main parameters and their ranges of change, leading to a decrease in the loss of valuable raw materials, increasing the productivity of the filters used, improving the quality of the output product, etc. when implementing the TPFS.

Based on the analysis of the numerical experiments carried out, conclusions are formulated that serve as the basis for making appropriate managerial decisions. medium.

Key words: mathematical model, numerical experiment, technological process, filtration, gel-particles, porous

Language: Russian

Citation: Ravshanov N, Saidov U, Shafiyev T (2018) DECISION OF THE DIRECT AND REVERSE PROBLEM OF THE TECHNOLOGICAL PROCESS OF SUSPENSION FILTERING. ISJ Theoretical \& Applied Science, 09 (65): 269-279.

Soi: http://s-o-i.org/1.1/TAS-09-65-45 Doi: crostef https://dx.doi.org/10.15863/TAS.2018.09.65.45

\section{РЕШЕНИЕ ПРЯМОЙ И ОБРАТНОЙ ЗАДАЧИ ТЕХНОЛОГИЧЕСКОГО ПРОЦЕССА ФИЛЬТРОВАНИЯ СУСПЕНЗИИ}

Аннотация: В статье рассматривается решение актуальной задачи, связанной с технологическим прочессом фильтрования и обезвоживания жидких растворов от мелкодисперсных частиц. Выше указанный технологический прочесс реализуется в ходе фильтрования и очистки химических растворов, питьевой воды, фармачевтических препаратов, жидкого топлива, продуктов общественного назначения и т.д. Для анализа, исследования, определения основных параметров технологического прочесса, а также режимов работь фильтровальных агрегатов и поддержки принятия управленческих решений была разработана математическая модель, где можно учитывать различных режимов работь фильтровального агрегата и физико-химических свойств растворов.

В статье отмечается, что исследование процесс фильтрования суспензии, определение основньх параметров рассматриваемого прочесса и их диапазонов изменения с иелью управления им на
\end{abstract}


функционирующих объектах затруднительно сбор данных о процессе занимает много времени, надо провести серию экспериментов в лабораторных условиях которые отнимают много рабочие силы и времени и трудно найти взаимосвязь между параметрами фильтра и технологчческого прочесса по ограниченной экспериментальной выборке.

Учитывая выше изложенного можно сказать, что в работе решается актуальная задача определение основных параметров и их диапазонов изменения приводящий к уменьшению потере ценного сырья, повышение производительности используемых фильтров, улучшение качества получаемого выходного продукта и т.д. при реализаџии ТПФС.

На основе анализа проведенных численных экспериментов сформулированы выводы, служащчие основанием для принятия соответствующих управленческих решений.

Ключевые слова: математическая модель, численный эксперимент, технологический процесс, фильтрование, гель-частийы, пористая среда.

\section{Введение}

Многочисленные отрасли производства заняты выделением отдельных составляющих многофазных и многокомпонентных смесей. В зависимости от физических и механических свойств составляющих, а также от масштаба обрабатываемого материала разработаны различные способы и устройства фильтрования и разделения отдельных составляющих смесей. Необходимостью увеличения производительности фильтровального агрегата и улучшения качества целевого продукта обусловлены усовершенствование этих устройств и подбор режимных показателей для каждого отдельного взятого процесса.

Фильтрование - эффективный метод разделения жидких неоднородных систем и растворов, широко применяемый в лабораторных и промышленных условиях (в химической, пищевой, нефтеперерабатывающей, горнорудной и других областях промышленности).

В технологии при фильтровании жидких растворов и суспензии используются различные по физико-механическим свойствам фильтры и фильтровальные перегородки. Работоспособность фильтрующего оборудования во многом определяется фильтрующими перегородками, с помощью которых осуществляется отделение частиц твердой фазы от жидкости или газа, гельчастиц, ионов от растворов и других. Фильтрующие перегородки, как правило, выполняются из пористых материалов в виде плоских или цилиндрических поверхностей, называемых фильтрующими элементами. Фильтрующие элементы обычно изготавливаются из ткани, керамики, металлокерамики, различного вида сеток, набора пластин, из волокнистых материалов и других, образующих фильтрующие проходные сечения заданной величины. Общим во всех перечисленных фильтрующих элементах является неизменность фильтрующих зазоров.

Как следует, из проведенных лабораторных экспериментов и анализов технологического процесса фильтрования смесей (ТПФС) в начальном этапе рассматриваемого процесса сопровождается некоторым периодом времени, в течение которого происходит образование слоя осадка над поверхности фильтрующими каналами агрегата. В этот период ТПФС неизбежен проскок твердых частиц, содержащихся в фильтруемой растворе. После формирования над каналами устойчивых фильтрующих слоев наступает период непосредственного фильтрования с получением чистого фильтрата на выходе фильтрующего агрегата. Чистота выходного раствора в этот период будет зависеть во первых от устойчивости фильтрующих сводов по отношению к воздействию давления фильтруемой суспензии; во вторых от устойчивости фильтрующих сводов по отношению к возможным вибрациям фильтрующей перегородки.

От величины пор фильтрующей перегородки зависят все стадии фильтрования: во-первых, время образования сводов, а, следовательно, и величина первоначального проскока твердых частиц суспензии; во-вторых, прочность образованных сводов; и, наконец, качество последующего установившегося процесса фильтрования. На этапе установившегося процесса фильтрования качество фильтрата (его чистота) зависит от пористости слоя, сформированного из задержанных частиц. Частицы, не задержанные слоем осадка, сумевшие проникнуть сквозь фильтрующие своды имеют возможность прилипнуть к внутренним поверхностям фильтрующих каналов за счет сил адгезии и уменьшить проходное сечение фильтрующей перегородки. Этот процесс прогрессирует с течением времени в случае использования пористой перегородки. Исходя из выше сказанного следует, что при фильтрование суспензии на технологический процесс содействует много параметров с различными весами. Отклонение этих параметров от нормы приводит к качественному и количественному изменениям рассматриваемого технологического процесса в целом. Поэтому определение основных параметров и их диапазонов изменения - один из основных вопросов в теории исследования и управления технологического процесса. 
Надо отметить, что исследование процесс фильтрования суспензии, определение основных параметров рассматриваемого процесса и их диапазонов изменения с целью управления им на функционирующих объектах затруднительно:

во-первых, сбор данных о процессе занимает много времени;

во-вторых, надо провести серию экспериментов в лабораторных условиях которые отнимают много рабочие силы и времени;

в-третьих, трудно найти взаимосвязь между параметрами фильтра и технологического процесса по ограниченной экспериментальной выборке.

Учитывая выше изложенного можно сказать, что в работа решается актуальная задача, выбор технологий и устройства и их режимы работы, а также определение основных параметров и их диапазонов изменения приводящий к уменьшению потере ценного сырья, повышение производительности используемых фильтров, улучшение качества получаемого выходного продукта и т.д. при реализации ТПФС.

Поэтому для достижения максимально лучшего результата по очистке конечного продукта от нежелательных примесей ТПФС должен быть организован как технологический цикл с оптимальными параметрами, включающими характеристики самих сепараторов и фильтров и режимы их работ.

В работе [1] предложена уравнение для описания процесса разделения промышленных суспензий в начальный период времени с целью исследование и установление экспериментальных закономерностей процессов разделения суспензий и обезвоживания получаемых осадков, а также выявление основных факторов, влияющих на производительность и эффективность работы промышленных фильтров и фильтровальных установок. Получены эмпирическая зависимость коэффициента Козени-Кармана от среднего диаметра частиц твёрдой фазы разделяемых концентратов и эмпирическое уравнение для кинетики обезвоживания осадков разделяемых суспензий, а также зависимость для определения величины расхода воздуха на вакуум-фильтрах при проведении процесса обезвоживания осадков на фильтровальных перегородках с учётом свойств обрабатываемой суспензии и её твёрдой фазы. В диссертационной работе Ширяева Е. В. Исследовано влияние удельного сопротивления слоя осадка на течение процессов фильтрования и обезвоживания осадков, разработать методы определения расходов воздуха, необходимых для обеспечения требуемой степени обезвоживания, а также для выбора комплектующего оборудования, а также процесс просушки осадков на фильтрах, разработать методики расчёта параметров процесса обезвоживания и определения наиболее рациональных режимов и условий проведения просушки на фильтре.

В работе [2] развита теоретические основы методов расчета гидромеханических процессов при фильтровании и центрифугировании суспензий с ньютоновской и неньютоновской реологией для широкого класса разделительного оборудования. Для достижения этой цели решается проблема, связанная с расчетом течения гетерогенных сред с твердой фазой.

Разработаны математические модели и проведены численные расчеты процесса фильтрования гетерогенных сред с образованием и без образования осадка при ее тонкослойном течении по проницаемым поверхностям произвольной формы с учетом начального участка, а также для процесса фильтрация суспензии по вращающимся проницаемым поверхностям произвольной формы.

Исследованы основные закономерностей движения дисперсных включений при течении гетерогенной среды с расслоением фаз под действием массовых сил, а также с учетом нелинейности ее реологического состояния. В многокритериальной постановке решена задача оптимизации работы барабанного вакуум-фильтра со сходящей рабочей лентой.

В статье [3] дано математическое описание процессов фильтрования суспензий, образовавшихся в процессе механической обработки пластин арсенида галлия, через металлические плоские пористые перегородки. Приведены формулы по математическому расчету процессов фильтрации суспензий через пористые металлические перегородки. Для разработки методов расчёта производственных установок проведён анализ зависимостей, приведённых в литературе и полученных в результате математической обработки экспериментальных данных.

В диссертационной работе Дьяченко Е. Н. [4] решены задачи связанные с нестационарным процессом фильтрования суспензии через пористых сред. Разработана статическая и динамическая модель формирования пористых сред. Получены научные результаты по поведению частиц и структур на микроуровне, которые были недоступны ранее в виду недостаточной вычислительной мощности ЭВМ и при использовании классических подходов механики сплошных сред. На основе метода дискретных элементов разработана численная модель фильтрования суспензии на насыпных фильтрах. Получены результаты моделирования фильтрования суспензии, показывающие пути увеличения производительности фильтров и оптимизации их работы. 
В [5] статье разработана математическая модель процесса фильтрации в производстве цинка, основанная на уравнениях материального баланса по расходам технологических сред и концентрациям отдельных компонентов. Модель позволяет определять расходы и количества получаемых продуктов процесса, оценивать его текущее состояние и прогнозировать будущие состояния.

В работе [6] рассматривается тупиковый режим фильтрации суспензии в канале. Такая ситуация характерна, например, для трубчатых и половоло-конных мембранных элементов и аппаратов с "тангенциальной" фильтрацией. Разработан численный модель процесса нестационарной фильтрации суспензий, позволяющей оптимизировать режимы работы фильтрационных установок и снизить затраты на научно-исследовательские и опытноконструкторские работы при создании промышленных установок. Для течения суспензии в канале принята модель вытеснения. На внутренней поверхности канала расположена фильтрующая перегородка, через которую проходит фильтрат за счет трансмембранного давления. Поток фильтрата зависит от гидравлических сопротивлений фильтрующей перегородки и слоя осадка на ее поверхности

В работе P.J.Monteiro, Ch.H.Rycroft и G.I.Barenblatt [7] разработана математическая модель фильтрация жидкости в нано-пористых породах. При выводе модели процесса предполагается, что фильтрационный слой состоит из двух компонентов: трещиноватопористая среда и специфические органические включения, состоящие из керогена, в основу которая положена гипотеза о том, что проницаемость включений существенно зависит от градиента давления.

B работе F.Boyer и др. [8] описывают некоторые аспекты моделирования диффузного потока несжимаемых сред, состоящих из трех несмешивающихся компонентов, без фазовых превращений, где при процессе моделирование трехфазных потоков дополнено путем включения уравнений Кана-Хилларда и Навье-Стокса. При этом поверхностное натяжение принимается во внимание через объемные капиллярные силы. Дискретизация уравнений выполняется по временной и пространственной переменным. Внимание авторов обращается на то, что большая часть основных свойств исходной модели, таких как сохранение объема и оценка энергии, должны быть сохранены на дискретном уровне. Для получения решения в случае очень тонких движущихся внутренних слоев используется адаптивный метод уточнения с ограничением общего количества узлов в сетке дискретной модели.

A.Raeini, M.Blunt и B.Bijeljic [9] представили стабильную численную схему для моделирования многофазного потока в пористых средах, когда характерный размер области течения составляет от микрона до миллиметров. Численный метод разработан для эффективного моделирования многофазных потоков в пористых средах со сложным характером движения границы раздела фаз и нерегулярных твердых границ. Уравнения Навье-Стокса дискретизированы с использованием подхода конечного объема, в то время как метод объема жидкости используется для нахождения расположений интерфейсов. Капилляные силы вычисляются с использованием модели полупроницаемой поверхностной силы, в которой область перехода для капиллярного давления эффективно ограничена одним узлом сетки.

М.А.Трапезникова, Н.Г.Чурбанова и А.А.Люпа [10] развивают математическую модель течения слабосжимаемой жидкости в пористой среде, построенной по аналогии с квазигазодинамической системой уравнений. Модель обобщена для случая трехфазной жидкости и дополнена уравнением сохранения энергии, что позволяет использовать ее при моделировании перспективных термических методов нефтедобычи.

Анонсируя выше изложенного можно сказать, что для комплексного исследования объекта, определения основных параметров и режимы работы фильтровальных агрегатов необходимо создать легко реализуемый инструмент. С этой точки зрения более подходящим методом решения поставленной задачи являются математическое моделирование (MМ) и вычислительный эксперимент (ВЭ) на основе анализа которых можно принять целесообразное решение.

Постановка задача. Математическая модель процесса фильтрования в безразмерной форме имеет вид [11-13]:

$$
\begin{gathered}
\frac{\partial W}{\partial t}+W \frac{\partial W}{\partial x}=-E u \frac{\partial P}{\partial x}+\frac{1}{\operatorname{Re}} \frac{\partial^{2} W}{\partial x^{2}}+\frac{W}{1-\theta} \frac{\partial \theta}{\partial t}-\frac{1}{\operatorname{Re}_{1}} \frac{W}{(1-\theta)(1-\delta)^{2}}, \\
\frac{\partial \theta}{\partial t}+\frac{1}{m} \frac{\partial W \theta}{\partial x}+\frac{\partial \alpha}{\partial t}+\left(1-m_{0}\right) \frac{\partial \delta}{\partial t}=\frac{\mu_{0} \alpha_{\tau}}{H_{0}^{2}} \frac{\partial}{\partial x}\left(\frac{\partial \theta}{\partial x}\right)^{2},
\end{gathered}
$$




\begin{tabular}{|c|c|c|c|c|c|c|}
\hline Impact Factor: & $\begin{array}{l}\text { ISRA (India) } \\
\text { ISI (Dubai, UAB } \\
\text { GIF (Australia) } \\
\text { JIF }\end{array}$ & $\begin{array}{r}=1.344 \\
=0.829 \\
=0.564 \\
=1.500\end{array}$ & $\begin{array}{l}\text { SIS (USA) } \\
\text { PИНЦ (Russia) } \\
\text { ESJI (KZ) } \\
\text { SJIF (Morocco) }\end{array}$ & $\begin{array}{l}=0.912 \\
=0.156 \\
=4.102 \\
=\mathbf{5 . 6 6 7}\end{array}$ & $\begin{array}{l}\text { ICV (Poland) } \\
\text { PIF (India) } \\
\text { IBI (India) }\end{array}$ & $\begin{array}{l}=6.630 \\
=1.940 \\
=4.260\end{array}$ \\
\hline
\end{tabular}

$$
\left.\begin{array}{c}
\frac{\partial \delta}{\partial t}=\lambda_{0}(\theta-\gamma \delta), \quad \theta=\frac{\alpha}{1-\delta}, \\
W=1 \quad \theta=e^{\frac{-\lambda m H_{0}\left(1-m_{0}\right) x}{W_{o}}}=\varphi(x), \quad \delta=0 \quad \text { ï đè } \quad t=0 \\
\frac{\partial W}{\partial x}=\frac{H_{0}^{3}}{H k_{0}}\left[P_{0}-\frac{W}{(1-\delta)^{2}}\right], \quad \theta=1 \quad \text { ï đè } \quad x=0 \\
\frac{\partial W}{\partial x}=0, \quad \frac{\partial \theta}{\partial x}=\frac{m H_{0} \lambda\left(1-m_{0}\right)}{W_{0}}\left(\gamma_{0} \delta-\theta\right), \text { ï đè } x=1
\end{array}\right\}
$$

где

$$
\begin{gathered}
\lambda_{0}=\lambda \alpha_{\tau}, \quad \gamma_{0}=\frac{\gamma \delta_{0}}{\theta_{0}}, \mathrm{P}_{0}=-\frac{k_{0}}{W_{0} H_{0} \mu} \frac{\partial P}{\partial x}, \\
R_{\ell}=\frac{\rho H_{0} W_{0}}{\mu}, \quad R_{\ell_{1}}=\frac{\rho H k_{0} W_{0}}{\mu H_{0}^{2}} \quad \text { - число Рейнольдса; } \\
P_{0}^{*}=\frac{\partial P}{\partial x}=\text { const }, \\
\mathrm{Eu}=\frac{P^{*}}{\rho W_{0}} \text { - число Эйлера }
\end{gathered}
$$

$W$ - скорость фильтрования; $\theta$ - объемная концентрация взвеси в движущей смеси; $\delta$ концентрация взвеси осевшей массы в порах фильтра; $\alpha$ - концентрация частиц, находящихся во взвешенном состоянии; $\rho$ и $\mu$ - плотность и вязкость суспензий; $P$ - давление в колонке агрегата; $\mathrm{H}, \mathrm{H}_{0}$ - соответственно, высота фильтровальной колонки агрегата и толщина фильтра; $k_{0}$ - коэффициент проницаемости фильтра до начала его работы; $\beta$ - эффективная константа обменивающихся ионов; $\chi$ коэффициент продольной диффузии; $\lambda$ кинетический коэффициент; $\gamma$ - коэффициент дисперсии; $\beta$ - эффективная константа скорости обменивающихся ионов; $m_{0}, \mathrm{~m}-$ начальная пористость и пористость фильтра.

Входные параметры $k_{0}, \lambda, \gamma$ являются опытными параметрами, которые определяются из лабораторных наблюдений. Однако, учитывая тот факт, что математическая модель является упрощенная и включает в себе член, полученный из обработке экспериментальных данных, то лабораторные данные этих параметров могут быть не пригодны для решения прямой задачи данного процесса. Поэтому, параметры математической модели целесообразно определять математически, т.е. из решения обратной задачи чем лабораторным.

Опытный параметр - коэффициент проницаемости имеет исключительно важное значение при расчетах ламинарного течения жидкости через пористую среду. Поэтому определение значения этого параметра в лабораторных условиях уделено много внимания. При выполнении этих работ удалено особое внимание зависимости его от размеров частиц грунта (фильтра) от вязкости жидкости и еe температуры и т.д. В работе [1] приведена методика определения проницаемости пористой среды при фильтрации газа и жидкости. Была установлено, что проницаемость определенная по газу, значительно выше проницаемости той же породы по жидкости. Кроме того было установлено, что проницаемости среды зависит от свойства жидкости. По мнению этих авторов различие в численном значении проницаемости породы при фильтрации жидкости и газа происходит в следствии физико-химических процессов взаимодействия жидкости с породой.

При лабораторных измерениях коэффициента проницаемости, из-за чрезвычайной сложности проведения эксперимента нередко допускаются ошибки (эти могут быть, как ошибки экспериментатора, так и погрешности приборов), которые приводят к неточному значению параметра. Следовательно, полученные численные значения прямой задачи с таким ошибочным параметром может привести к несоответствию результатов математической модели с экспериментальном данными и данными производства. С этой точки зрения проведение дополнительных расчетов с использованием уже других опытных дынных с математической моделью, т.е. решение обратной задачи представляет особый интерес.

Если существуют опытные данные $W\left(t_{j}\right)$ при $\Delta P=$ const или $\Delta P\left(t_{j}\right)$ при $W=$ const, то решая обратную задачу, можно определить численные значение параметров $k_{0}, \lambda, \gamma$. Нужно отметить, что получить эти опытные данные проще чем определение параметров. Например, в самом фильтровальной колонке, работающей при $W=$ const установлен прибор, который фиксирует, изменения давление на специальной бумажной диаграмме (Ферганский завод искусственного химического волокна). Эти 


\begin{tabular}{|c|c|c|c|c|c|c|}
\hline Impact Factor: & $\begin{array}{l}\text { ISRA (India) } \\
\text { ISI (Dubai, UAE } \\
\text { GIF (Australia) } \\
\text { JIF }\end{array}$ & $\begin{array}{l}=1.344 \\
=0.829 \\
=0.564 \\
=1.500\end{array}$ & $\begin{array}{l}\text { SIS (USA) } \\
\text { PИНЦ (Russia) } \\
\text { ESJI (KZ) } \\
\text { SJIF (Morocco) }\end{array}$ & $\begin{array}{l}=0.912 \\
=0.156 \\
=4.102 \\
=5.667\end{array}$ & $\begin{array}{l}\text { ICV (Poland) } \\
\text { PIF (India) } \\
\text { IBI (India) }\end{array}$ & $\begin{array}{l}=6.630 \\
=1.940 \\
=4.260\end{array}$ \\
\hline
\end{tabular}

диаграммы можно использовать при решении обратной задачи

$$
\begin{gathered}
\frac{1}{k_{0}(1-\delta)^{2}}=\frac{1}{k_{0}^{(s-1)}\left(1-\delta^{(s-1)}\right)^{2}}-\frac{k_{0}^{s}-k_{0}^{(s-1)}}{\left(k_{0}^{(s-1)^{2}}\right)^{2}\left(1-\delta^{(s-1)}\right)^{2}}-\frac{2}{k_{0}^{(s-1)}\left((1-\delta)^{(s-1)}\right)^{3}} \bullet \\
\bullet \frac{\partial \delta^{(s-1)}}{\partial \gamma}\left(\lambda^{(s)}-\lambda^{(s-1)}\right)-\frac{2}{k_{0}^{(s-1)}\left(\left(1-\delta^{(s-1)}\right)\right)^{3}} \frac{\partial \delta^{(s-1)}}{\partial \gamma}\left(\gamma^{(s)}-\gamma^{(s-1)}\right), \\
\frac{\partial \delta}{\partial \lambda}=\theta_{0} e^{-\left(B x+t \gamma^{(s-1)} \lambda^{(s-1)}\right)}\left[\left(\frac{B x \lambda^{(s-1)}}{2 \gamma^{(s-1)}}-\frac{1+t}{\gamma^{(s-1)}}\right)\right] \cdot\left(B x+t \gamma^{(s-1)} \lambda^{(s-1)}\right)-\frac{B x}{\lambda \gamma^{(s-1)}}, \\
\frac{\partial \delta^{(s-1)}}{\partial \lambda}=\lambda^{(s-1)} e^{-B x \lambda^{(s-1)}}\left(\frac{B x}{2}-\frac{1}{\lambda^{(s-1)}}\right) \cdot \frac{1}{\gamma^{(s-1)}} \cdot\left[\frac{1}{\gamma^{(s-1)}}-e^{-(\lambda \gamma)^{(s-1)}}\left(\lambda^{(s-1)} t+\frac{1}{\gamma^{(s-1)}}\right)\right],
\end{gathered}
$$

где

$$
B=\frac{1-m_{0}}{W_{0}}
$$

Математическая моделью для обратной задачи могут служить уравнения (2)-(3). Интегрируя это уравнения в переделах от 0 до $H_{0}$, получим:

$$
P\left(t_{j}\right)-P_{0}=\frac{\mu H_{0} W_{0}}{H k_{0}} \int_{0}^{H_{0}} \frac{d x}{(1-\delta)^{2}}-\left.\rho H_{0} W_{0} \frac{\partial \theta}{\partial x}\right|_{x=H_{0}}
$$

Тогда параметры могут быть определены из минимума функционала

$$
R=\int_{0}^{t}\left|P\left(\tau, k_{0}, \lambda, \gamma\right)-\tilde{P}(\tau)\right|^{2} d \tau=\min
$$

Функции $\delta$ в (5) можно вычислить из решение уравнение баланса и кинетики Ю.М.Шехтмана, полученное им в работе [14]. Для этого разложим функцию $I_{0}\left(2 \sqrt{\lambda^{2} \gamma B x t}\right)$ входящую в ряд, ограничиваясь двумя членами и выполняя операцию интегрирования, получим

$\delta(x, t)=\theta_{o} \lambda e^{-\lambda B x}\left[e^{-\lambda B t}\left(\frac{B x}{\partial \gamma}-\frac{1+f}{\lambda \gamma}\right)+\frac{1}{\lambda \gamma}-\frac{B x}{\lambda \gamma}\right]$,

Тогда параметры можно определить

из системы алгебраических уравнений

$$
\left.\begin{array}{l}
\frac{\partial R}{\partial k_{0}}=0, \\
\frac{\partial R}{\partial \lambda}=0, \\
\frac{\partial R}{\partial \gamma}=0
\end{array}\right\}
$$

Методы решения задача. Система уравнения (8) является нелинейной относительно неизвестных $k_{0}, \lambda, \gamma$ решение которой связанной с определенными трудностями. Однако, применяя метод квазилинеаризации [15] к (5), (7) можно свести к линейной системе. Для этого предполагая, что все условия теоремы дифференцирование под знаком интегралом выполнены, подставляя эти значения в (5) получим:

$$
P(t)=f_{1}+f_{2} k_{0}+f_{3} \lambda+f_{4} \gamma
$$

где

$$
\begin{aligned}
& f_{i}=f_{i}\left(t, k_{0}^{s-1}\right), \quad \lambda^{(s-1)}, \quad \gamma^{(s-1)}, \\
& \delta^{(s-1)}=\delta\left(x, t, \lambda^{(s-1)}\right), \quad \gamma^{(s-1)},
\end{aligned}
$$

Таким образом, из системы (8) можно определить искомые параметры.

Основные теоремы устойчивости обратные задач впервые сформулированы и доказаны в работах А.Н. Тихонова [15].

Решение обратной задачи в данной случае заключается в том, что зная приближенные значения или совершенно не зная их, определить такое численное значение, чтобы оно адекватно соответствовало математические модели с производственными процессами. А без наличии указанных «оценок» теряет определенный смысл само решения задачи и решаемых на ее основе.

Поскольку $\tilde{P}_{j}=\tilde{P}\left(t_{j}\right)$ получены из опыта, то очевидно они содержат некоторые погрешности (это могут быт вибрации аппарата, ошибки при чтении данных из диаграммы, 


\begin{tabular}{|c|c|c|c|c|c|c|}
\hline Impact Factor: & $\begin{array}{l}\text { ISRA (India) } \\
\text { ISI (Dubai, UAE } \\
\text { GIF (Australia) } \\
\text { JIF }\end{array}$ & $\begin{array}{r}=1.344 \\
=0.829 \\
=0.564 \\
=1.500\end{array}$ & $\begin{array}{l}\text { SIS (USA) } \\
\text { PИНЦ (Russia) } \\
\text { ESJI (KZ) } \\
\text { SJIF (Morocco) }\end{array}$ & $\begin{array}{l}=0.912 \\
=0.156 \\
=4.102 \\
=5.667\end{array}$ & $\begin{array}{l}\text { ICV (Poland) } \\
\text { PIF (India) } \\
\text { IBI (India) }\end{array}$ & $\begin{array}{l}=6.630 \\
=1.940 \\
=4.260\end{array}$ \\
\hline
\end{tabular}

человеческий фактор при ввода полученных данных в вычислительной машины и т.д.).

Если опытные данные $P_{j}$ подставить в (6), а интеграл заменить суммой, то вообще говоря, получится способ для определения $k_{0}, \lambda$ ѐ $\gamma$. Но такой метод определения параметров, как указано в работах многих авторов, будет неустойчивым, т.е. для определения поправок может получиться плохо обусловленная линейная система. Для решения задача в такой постановке можно применяя регуляризацию А.Н. Тихонова [15] и можно решить задачу до конца.

Однако, для массовой обработки опытных данных, с достаточной для практики точностью, можно получить устойчивый метод решения обратных задач, если пользоваться следующей методикой.

Опытные данные $\tilde{\mathrm{P}}_{j}$, заданные таблично, аппроксимируются полиномом Чебышева. Оценки дисперсий аппроксимирующих полиномов степени $n$ и $n+1$ обозначим через $S_{n}$ и $S_{n+1}$ соответственно. Если $S_{n}>S_{n+1}$, то степен предпочтительнее, то для принятия окончательного решения необходимо использовать критерий Фишера [16], т.е., если

$$
\frac{S_{n}}{S_{n+1}}>F_{v}\left(\bar{\psi}_{1}, \bar{\psi}_{2}\right)
$$

( $v$ - уровень значимости; $\bar{\psi}_{1}$ ѐ $\bar{\psi}_{2}-$ степенны свободы), то степень аппроксимирующего полинома принимается равной $n+1$, в противной случае $-n$. Повторяя этот процесс для $n=1,2 \ldots \ldots .$. находим степень аппроксимирующего полинома.

Аппроксимирующий

полином представляется в виде:

$$
P(t)=\sum_{k=1}^{n} a_{k} \varphi_{k}(t)
$$

где

$$
a_{k}=\frac{\sum_{i=1}^{m} \tilde{P}_{i} \varphi_{k}\left(t_{i}\right)}{\sum_{i=1}^{m} \varphi_{k}^{2}\left(t_{i}\right)}
$$

$\varphi_{k}(t)$ - полином Чебышева $k$-ой степени; $a_{k}$ коэффициент аппроксимирующего полинома, который определяется по известным рекуррентным соотношениям.

Оценка дисперсий аппроксимирующего полинома вычисляется с помощью соотношения

$$
S=\frac{\sum_{i=1}^{m}\left(\tilde{P}_{i}-P_{i}\right)}{m-(n-1)}
$$

После получения аппроксимирующего полинома вычисляется корреляционное соотношение

$$
\begin{gathered}
\eta=\sqrt{1-\zeta_{k}}, \\
\text { где } \\
\zeta_{k}=\frac{r_{n}}{r_{0}} ; \quad r_{n}=S_{n}(m-1) ; \\
r_{0}=\sum_{i=1}^{m} \tilde{P}_{i}^{\lambda}-\left(\sum_{i=1}^{m} \tilde{P}_{i}\right)^{\lambda} .
\end{gathered}
$$

Корреляционное

соотношение $\quad \eta$ характеризует тесноту аппроксимирующего полинома и опытных данных. Если $\eta$ близко к 1, то теснота хорошая. В частности, если $\eta=1$, то аппроксимирующего полином должен во всех точках опытных данных. Поэтому всегда должно выполняться условие

$$
0<\eta<1 \text {. }
$$

Подставляя в (6) вместо опытных данных $\tilde{P}_{j}$ полиномы Чебышева, получим

$$
R=\int_{o}^{t}[P(\tau)-\varphi(\tau)]^{2} d \tau=\min
$$

или

$$
R=\sum_{i=1}^{m}\left[P_{n_{i}}+\sum_{i=1}^{m} \frac{\partial P_{n i}}{\partial \alpha_{\ell}}-\varphi_{i}\right]^{2}=\min
$$

здесь, учитывая непрерывную зависимость $P$ от параметров, предварительно разложено последнее выражение в ряд Тейлора

$$
\begin{gathered}
P_{n+1}=P_{n}+\sum_{l=1}^{3} \frac{\partial P_{n}}{\partial \alpha_{\ell}}+0\left(\alpha_{\ell}^{\lambda}\right) ; \\
P_{n_{i}}=P_{n}\left(t_{i}\right)=P\left(t_{i}, k_{0}, \lambda, \gamma\right) ; \\
\frac{\partial P}{\partial k_{0}}=\frac{\partial P\left(t_{i}\right)}{\partial k_{0}} ; \quad \frac{\partial P}{\partial \lambda}=\frac{\partial P\left(t_{i}\right)}{\partial \lambda} ; \frac{\partial P}{\partial \gamma}=\frac{\partial P\left(t_{i}\right)}{\partial \gamma} ; \\
\varphi_{i}=\varphi\left(t_{i}\right)=\sum_{l=1}^{m} \alpha_{l} \varphi_{e}\left(t_{i}\right) .
\end{gathered}
$$

Таким образом, параметры

$k_{0}, \lambda$ è $\gamma$ находятся из системы алгебраических уравнений (8), решение которой получить не представляет труда. Итерационный процесс продолжается до выполнения условия

$$
\max _{\ell}\left|\alpha_{\ell}\right|<\varepsilon, \quad \varepsilon>0
$$

( $S$-номер итерации).

Вычислительный процесс провидится по следующей схеме:

- Задаются

начальные

значения

$k_{o}^{(0)}, \lambda^{(0)}, \gamma^{(0)}$

вычисляется

значение 


\begin{tabular}{|c|c|c|c|c|c|c|}
\hline Impact Factor: & $\begin{array}{l}\text { ISRA (India) } \\
\text { ISI (Dubai, UAE } \\
\text { GIF (Australia) } \\
\text { JIF }\end{array}$ & $\begin{array}{l}=1.344 \\
=0.829 \\
=0.564 \\
=1.500\end{array}$ & $\begin{array}{l}\text { SIS (USA) } \\
\text { PИНЦ (Russia) } \\
\text { ESJI (KZ) } \\
\text { SJIF (Morocco) }\end{array}$ & $\begin{array}{l}=0.912 \\
=0.156 \\
=4.102 \\
=5.667\end{array}$ & $\begin{array}{l}\text { ICV (Poland) } \\
\text { PIF (India) } \\
\text { IBI (India) }\end{array}$ & $\begin{array}{l}=6.630 \\
=1.940 \\
=4.260\end{array}$ \\
\hline
\end{tabular}

- Затем, из (2.32) находятся значения $k_{o}^{(1)}, \lambda^{(1)}, \gamma^{(1)}$;

- Проверяется условие (9). В случае не выполнения, за начальное значения параметров принимая уже найденные значения $k_{o}^{(1)}, \lambda^{(1)}, \gamma^{(1)}$, находятся из (8) $k_{o}^{(2)}, \lambda^{(2)}, \gamma^{(2)}$, и т.д. При выполнении условия (9) на итерации, за искомые параметры принимаются значения $k_{o}^{(N)}, \lambda^{(N)}, \gamma^{(N)}$.

По изложенной схеме были произведены расчеты по следующими исходными данными: $W_{0}$ $=0,0025 \mathrm{м} /$ сек; $m_{1}=0,4 ; m_{0}=0,15 ; H_{0}=0,5 \mathrm{м}$.; $\rho=25$ г/см.; $H=1$ м.; $\mu=980$ спуаз.

Анализ результатов и выводы. На основе выше изложенного алгоритма проведены численные расчеты на ЭВМ, результат которых представлены на рис 1-
На рис. 1-4 построены кривые по этим исходным и соответствующие им аппроксимирующие полиномы по Чебышеву.

Анализ полученных результаты проведенных численных расчетов (рис. 1-4) и сопоставление их с экспериментальными данными показали, что вычисление истинные значение параметров технологического процесса играет существенный роль при моделирование объекта исследования, а также определения их основных показателей со временим. Как показали проведенных численных расчетов на ЭВМ с ростом вычисления истинного значения параметров процесса степень адекватности разработанного ММ объекта повышается (рис. 1-4). Эта можно особенно заметить при сопоставление результатов полученные с модельными и экспериментальными данными (рис. 4).

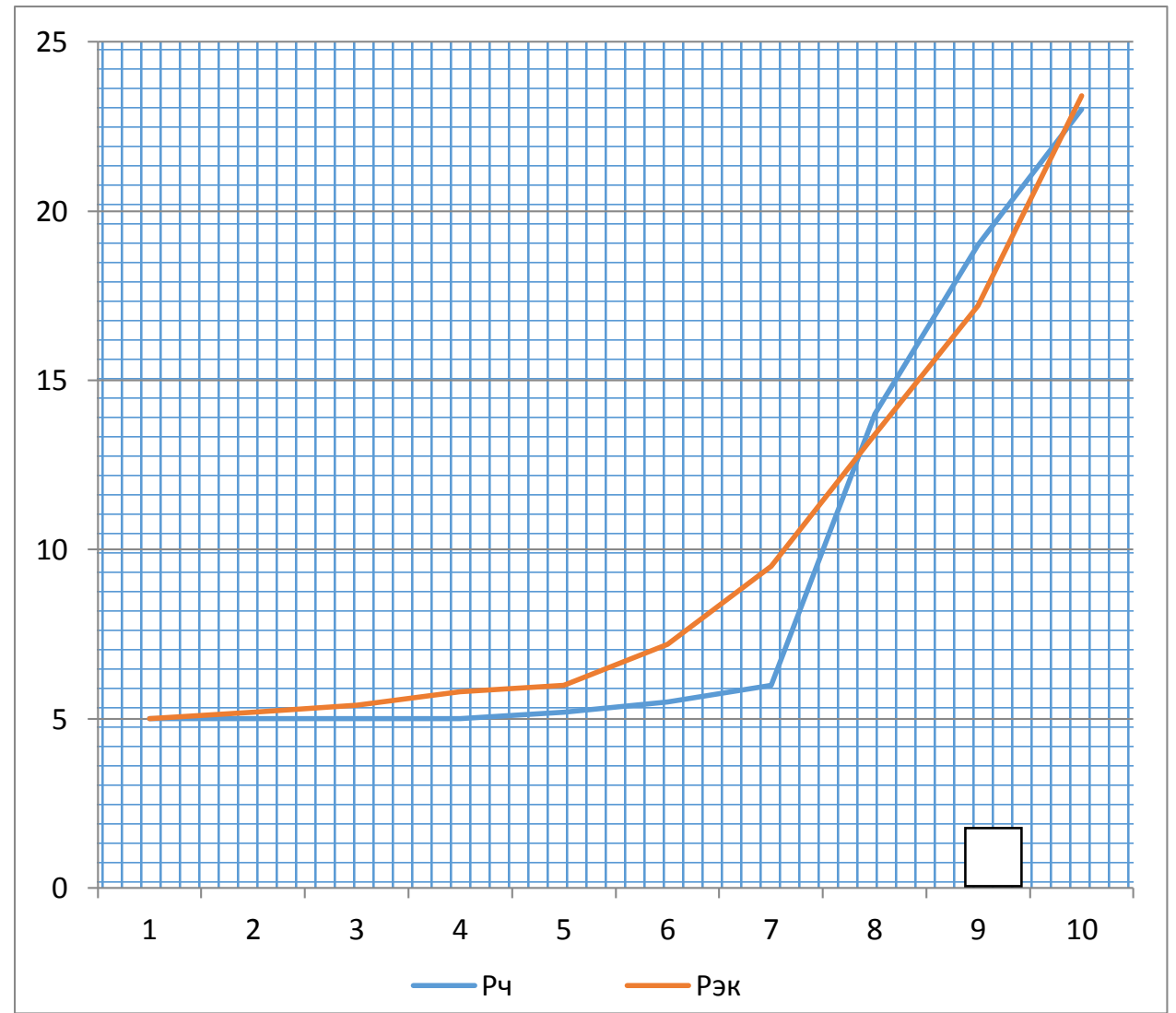

Рис. 1. Рост изменения давления внутри фильтровальной колонки агрегата при $k_{0}=9,6$ Дарси; $\lambda \cdot 10^{-3}=0,241 /$ cek; $\gamma=0,004$ 


\begin{tabular}{|c|c|c|c|c|c|c|}
\hline Impact Factor: & $\begin{array}{l}\text { ISRA (India) } \\
\text { ISI (Dubai, UAE } \\
\text { GIF (Australia) } \\
\text { JIF }\end{array}$ & $\begin{array}{l}=1.344 \\
=0.829 \\
=0.564 \\
=1.500\end{array}$ & $\begin{array}{l}\text { SIS (USA) } \\
\text { PИНЦ (Russia) } \\
\text { ESJI (KZ) } \\
\text { SJIF (Morocco) }\end{array}$ & $\begin{array}{l}=0.912 \\
=0.156 \\
=4.102 \\
=\mathbf{5 . 6 6 7}\end{array}$ & $\begin{array}{l}\text { ICV (Poland) } \\
\text { PIF (India) } \\
\text { IBI (India) }\end{array}$ & $\begin{array}{l}=6.630 \\
=1.940 \\
=4.260\end{array}$ \\
\hline
\end{tabular}

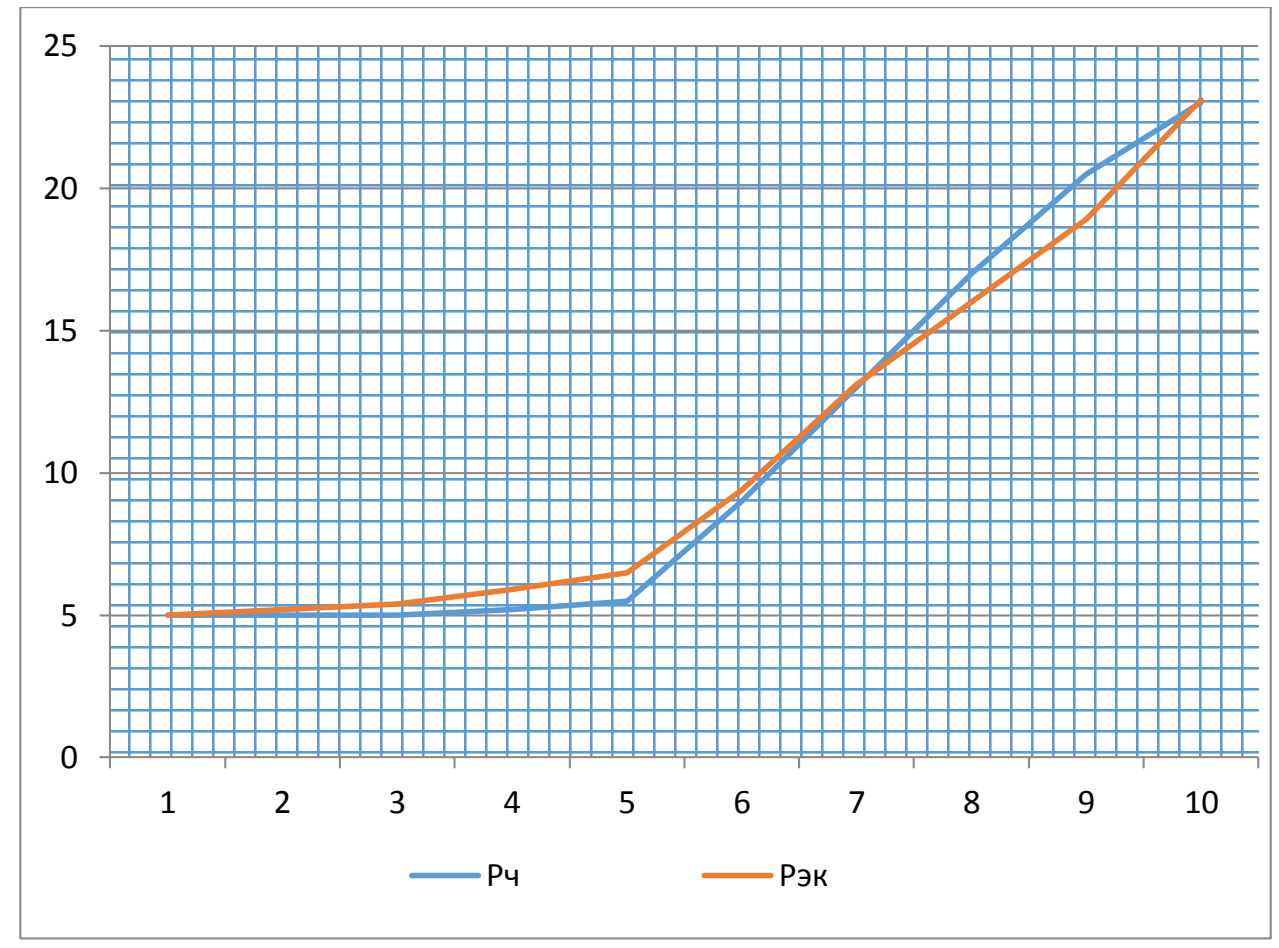

Рис. 2. Рост изменения давления внутри фильтровальной колонки агрегата при $k_{0}=9,82$ Дарси; $\lambda \cdot 10^{-3}=0,241 / c e k ; \gamma=0,004$

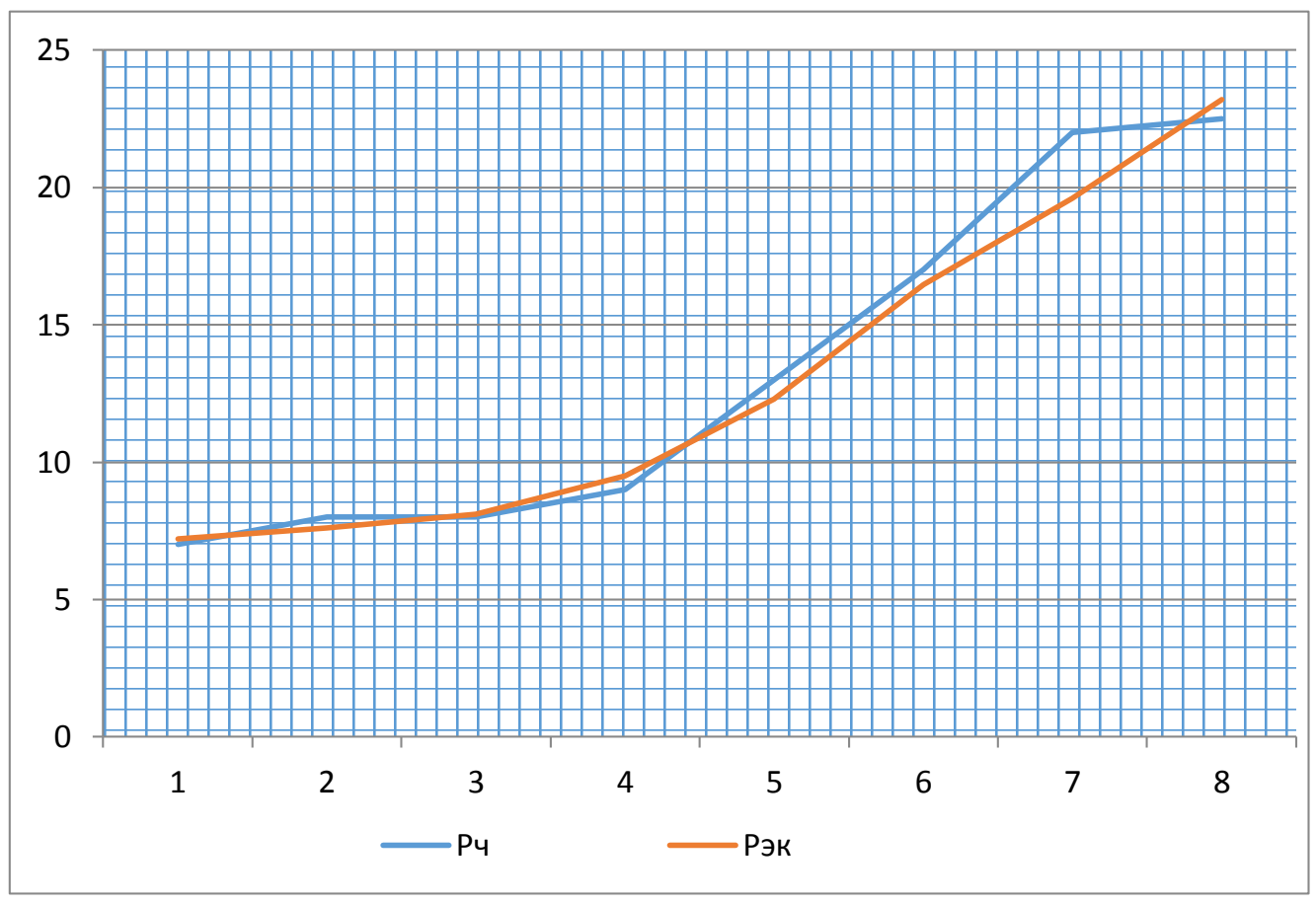

Рис. 3. Рост изменения давления внутри фильтровальной

колонки агрегата при $k_{0}=8,7$ Дарси; $\lambda \cdot 10^{-3}=0,211 /$ cek; $\gamma=0,47$ 


\begin{tabular}{|c|c|c|c|c|c|c|}
\hline Impact Factor: & $\begin{array}{l}\text { ISRA (India) } \\
\text { ISI (Dubai, UAE } \\
\text { GIF (Australia) } \\
\text { JIF }\end{array}$ & $\begin{array}{r}=1.344 \\
=0.829 \\
=0.564 \\
=1.500\end{array}$ & $\begin{array}{l}\text { SIS (USA) } \\
\text { PИНЦ (Russia) } \\
\text { ESJI (KZ) } \\
\text { SJIF (Morocco) }\end{array}$ & $\begin{array}{l}=0.912 \\
=0.156 \\
=4.102 \\
=5.667\end{array}$ & $\begin{array}{l}\text { ICV (Poland) } \\
\text { PIF (India) } \\
\text { IBI (India) }\end{array}$ & $\begin{array}{l}=6.630 \\
=1.940 \\
=4.260\end{array}$ \\
\hline
\end{tabular}

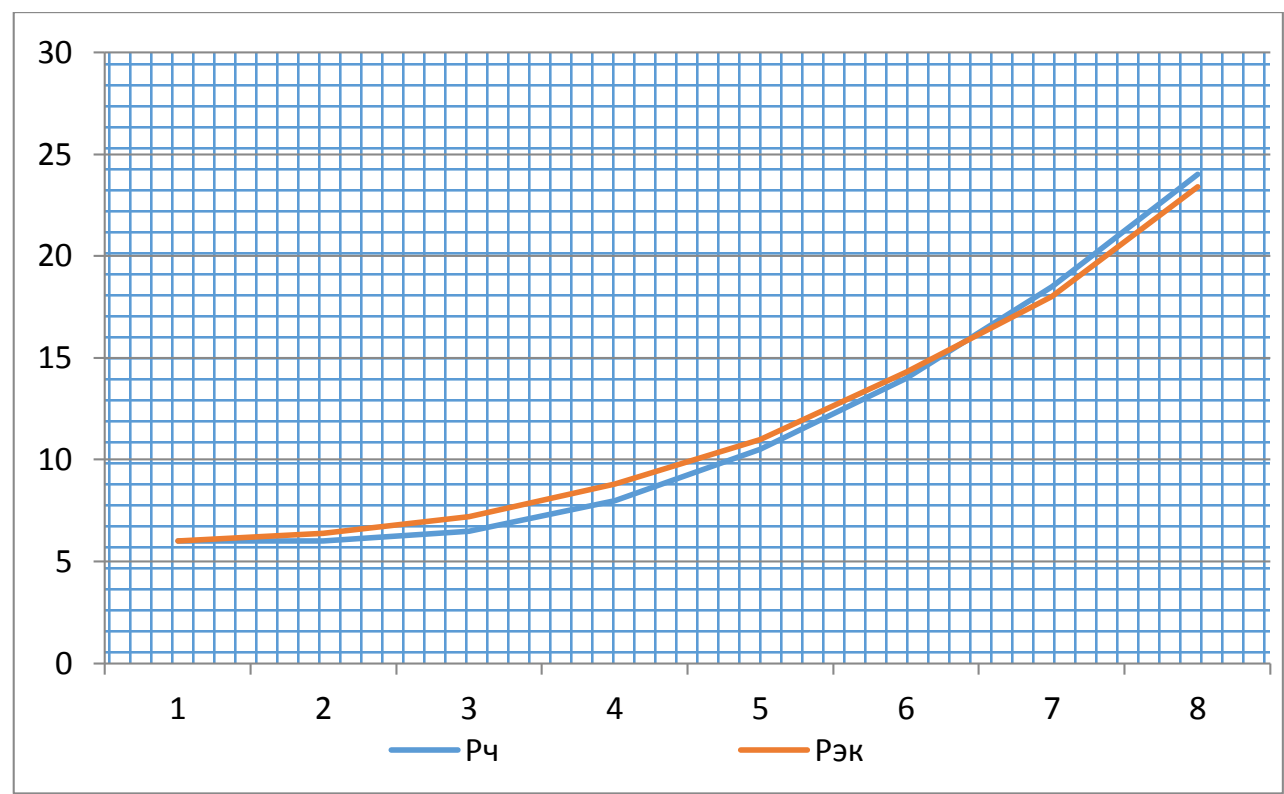

Рис. 4. Рост изменения давления внутри фильтровальной

колонки агрегата при $k_{0}=9,0$ Дарси; $\lambda \cdot 10^{-3}=0,211 / c e k ; \gamma=0,0043$

И так можно утверждать, что решая обратную задачи и подставляя найденные значения параметров в исходную систему уравнений вычисляются искомые функции $W, \delta, \theta$.

\section{Выводы.}

Во первых, в ходе проведенных исследование утверждено, что полученные из обработке экспериментальных данных, то ест лабораторные данные входные параметры $k_{0}, \lambda, \gamma$ не пригодны для решения прямой задачи процесса. Поэтому, параметры математической модели целесообразно определять математически, т.е. из решения обратной задачи чем лабораторным путем (рис.4). Во вторых, адекватность разработанного ММ объекта можно достичь за счет вычисления точного значения параметров рассматриваемого ТПФС.

\section{References:}

1. Shiryaeva, Elena Vasil'evna (2011) Protsessy fil'trovaniya suspenziy i obezvozhivaniya osadkov na promyshlennykh vakuumfil'troval'nykh ustanovkakh nepreryvnogo deystviya dissertatsiya ... kandidata tekhnicheskikh nauk: 05.17.08- Moskva, 2011, $156 \mathrm{p}$.

2. Ibyatov Ravil' Ibragimovich. (2005) Metody rascheta gidromekhanicheskikh protsessov pri fil'trovanii i tsentrifugirovanii suspenziy : : 05.17.08.- Kazan', 2005.- 340 p.

3. Akul'shin A.A., Bredikhina N. V., Nozdratenko S. A. (2016) Matematicheskoe opisanie protsessa fil'trovaniya suspenziy cherez metallicheskie ploskie poristye peregorodki //
Izvestiya Yugo-Zapadnogo gosudarstvennogo universiteta. - 2016. - № 6(69);

4. (2018) URL: iswsu.esrae.ru/26-120 (data obrashcheniya: 15.05 .2018$)$. 22-30 p.

5. D'yachenko E. N. (2010) Chislennoe modelirovanie poristykh struktur i fil'trovaniya suspenzii metodom diskretnykh elementov, dissertatsiya ... kandidata kandidata fizikomatematicheskikh nauk: 01.02.05. - Tomsk2010, 126 p.

6. Rutkovskiy A.L., Dyunova D.N. (2014) Razrabotka i issledovanie matematicheskoy modeli protsessa fil'tratsii v proizvodstve tsinka. Izvestiya vuzov. Tsvetnaya metallurgiya. 


\begin{tabular}{l|lr|ll|ll} 
& ISRA (India) & $=\mathbf{1 . 3 4 4}$ & SIS (USA) & $=\mathbf{0 . 9 1 2}$ & ICV (Poland) & $=\mathbf{6 . 6 3 0}$ \\
Impact Factor: & ISI (Dubai, UAE) $=\mathbf{0 . 8 2 9}$ & PUHЦ (Russia) $=\mathbf{0 . 1 5 6}$ & PIF (India) & $=\mathbf{1 . 9 4 0}$ \\
& GIF (Australia) & $\mathbf{0 . 5 6 4}$ & ESJI (KZ) & $=4.102$ & IBI (India) & $=\mathbf{4 . 2 6 0}$ \\
& JIF & $=\mathbf{1 . 5 0 0}$ & SJIF (Morocco) & $=2.031$ & & \\
\hline
\end{tabular}

2014;(6): p. 62-66. DOI:10.17073/0021-34382014-6-62-66

7. Davydova E. B., Il'in M. I., Tarasov A. V. (2013) Modelirovanie nestatsionarnogo protsessa fil'tratsii suspenziy $\mathrm{v}$ tupikovom kanale// Teoreticheskie osnovy khimicheskoy tekhnologii, 2013, tom 47, № 3, p. 352-354

8. Monteiro P.J., Rycroft Ch.H., Barenblatt G.I. (2012) A mathematical model of fluid and gas flow in nanoporous media // Proceedings of the National Academy of Sciences of the United States of America. - 2012. - vol. 109, № 50. p. 20309-20313.

9. Boyer F., Lapuerta C., Minjeaud S., Piar B., Quintard M. (2010) Cahn-Hilliard/NavierStokes Model for the Simulation of ThreePhase Flows // Transport in Porous Media. 2010. - vol. 82, № 3. - Rp. 463-483. doi:10.1007/s11242-009-9408-z.

10. Ali Q. Raeini, Martin J. Blunt, Branko Bijeljic (2012) Modelling two-phase flow in porous media at the pore scale using the volume-offluid method // Journal of Computational Physics.-USA, 2012. - vol. 231, № 17. - Pp. 5653-5668. doi>10.1016/j.jcp.2012.04.011.

11. Trapeznikova M.A., Churbanova N.G., Lyupa A.A. (2015) Modelirovanie techeniya trekhfaznoy zhidkosti $\mathrm{V}$ poristoy srede $\mathrm{S}$ uchetom termicheskikh effektov // Mathematica Montisnigri. - 2015. - vol. 33. - p. 105-115.

12. 11. Ravshanov N., Palvanov B.Yu. (2017) Matematicheskaya model' dlya ionoobmennoy sorbtsii dvukhkomponentnykh rastvorov // Problemy vychislitel'noy i prikladnoy matematiki. - Tashkent, 2017. - № 5 (11). p. 55-61 (05.00.00; №23).

13. Ravshanov N., Palvanov B.Yu. (2017) Numerical solution of inverse problems filtering process of low-concentration solutions // ISJ Theoretical \& Applied science. - 2017. № 04 (48). - p. 85-94.

14. Ravshanov N., Palvanov B.Yu. (2012) Obratnaya zadacha dlya opredeleniya parametrov ionoobmennogo fil'trovaniya suspenzii // Voprosy vychislitel'noy i prikladnoy matematiki: Sb. nauchn. tr. Tashkent, 2012. - Vyp. 127. - p. 87-108.

15. Tikhonov A. N., Arsenin V. Ya. (1979) Metody resheniya nekorrektnykh zadach. - M.: Nauka, 1979. - 283 p.

16. R.A. Fisher. (2018) Statistical methods for research workers by [electronic resource] - 7th ed., rev. and enl. Edinburgh Oliver and Boyd Biological monographs and manuals, Xv, 356 p. 